\title{
Evaluating Mechanisms: Mapping Weekly Dynamics between Experiential Avoidance and SAD Symptoms
}

\author{
Laurel D. Sarfan $^{1,2} \cdot$ Joshua C. Magee ${ }^{1} \cdot$ Elise M. Clerkin $^{1}$
}

Accepted: 25 May 2021 / Published online: 25 June 2021

(c) The Author(s) 2021

\begin{abstract}
Widely-used, empirically-supported treatments focus on reducing experiential avoidance (EA) as a mechanism of social anxiety disorder (SAD) symptom change. However, little is known about how EA and SAD symptoms bidirectionally interrelate from session to session, or throughout the course of an intervention-a gap that raises significant theoretical and clinical questions about the mechanistic role of EA. Participants $(N=78)$ with elevated EA and SAD symptoms completed a 3-session pilot intervention (Approach-Avoidance Task training plus psychoeducation) designed to target EA. Bivariate latent change score modeling was then used to map the bidirectional, temporal interrelationships between EA and SAD symptoms from session to session. Analyses accounted for the overall trajectory of change in both variables (i.e., EA and SAD) and both variables' preceding measurement. Findings suggested that changes in SAD symptoms preceded and predicted changes in EA from session to session. Contrary to hypotheses, this effect was not bidirectional, as changes in EA did not precede and predict changes in SAD symptoms from session to session. The use of a relatively small analogue sample limit the external validity of the present findings. Nevertheless, these novel findings advance our understanding of the dynamic interrelationships between EA and SAD symptoms throughout treatment. Moreover, given that many leading treatments target EA, this study highlights a need for future work to continue evaluating whether EA is indeed a mechanism of SAD symptom change.
\end{abstract}

Keywords Experiential avoidance $\cdot$ Social anxiety disorder $\cdot$ Mechanisms $\cdot$ Bivariate latent change score modeling . Approach-avoidance task training $\cdot$ Psychoeducation

$\begin{array}{ll}\text { Abbreviations } \\ \text { AAQ } & \text { Acceptance and action questionnaire } \\ \text { AAT } & \text { Approach-avoidance task } \\ \text { BEAQ } & \text { Brief experiential avoidance questionnaire } \\ \text { BLCSM } & \text { Bivariate latent change score modeling } \\ \text { CEQ-R } & \text { Credibility/expectancy questionnaire - revised } \\ \text { CFI } & \text { Comparative fit index } \\ \text { EA } & \text { Experiential avoidance } \\ \text { RMSEA } & \text { Root mean square error of approximation }\end{array}$

Laurel D. Sarfan

sarfanld@berkeley.edu

Joshua C. Magee

mageejc@miamioh.edu

Elise M. Clerkin

clerkiem@miamioh.edu

Miami University, Oxford, OH 45056, USA

2 Present Address: University of California, Berkeley, 2121 Berkeley Way, Berkeley, CA 94720, USA
SAD Social anxiety disorder

SPIN Social Phobia Inventory

\section{Introduction}

Social anxiety disorder, or SAD, is one of the most prevalent lifetime disorders in the United States (Kessler et al., 2005 , 2011). Fear of negative evaluation is thought to be the core feature of SAD, which can result in debilitating anxiety and avoidance of social situations (Heimberg et al., 2014). Although years have been spent developing treatments to target SAD, full recovery rates are often low (Hofmann \& Bögels, 2006; Steinert et al., 2013); thus, more research is needed to evaluate the mechanisms driving this disorder.

\section{Experiential Avoidance}

Experiential avoidance, or EA, has been identified as one potential mechanism driving SAD (Glick \& Orsillo, 2011; Kashdan et al., 2006, 2014). EA is a transdiagnostic emotion 
regulation strategy characterized by internal resistance to and avoidance of uncomfortable thoughts, emotions, and physical sensations (Chawla \& Ostafin, 2007; Hayes et al., 1996). Several studies have found that individuals diagnosed with SAD (Kashdan et al., 2013) and with high SAD symptoms (Mahaffey et al., 2013; O'Toole et al., 2017) report greater use of EA compared to healthy controls or individuals with low SAD symptoms. Further, findings from a number of randomized control trials suggest that EA may play a meaningful role in treatment outcomes for SAD symptoms (Dalrymple \& Herbert, 2007; Niles et al., 2014; Ossman et al., 2006).

Despite this compelling body of literature, little is known about how EA and SAD symptoms temporally interrelate throughout treatment. This dearth of research is notable given the theoretical and clinical emphasis on EA in leading empirically-supported treatments. For example, Acceptance and Commitment Therapy (Hayes et al., 2006), the Unified Protocol (Barlow et al., 2004), and Acceptance-Based Behavior Therapy (Roemer et al., 2008) each emphasize reducing EA via targeted modules for psychopathology, including those for anxiety disorders. Yet, past research has not dismantled whether targeting EA directly impacts changes in SAD symptoms from session-to-session.

Evaluating these session-by-session interrelationships throughout treatment may be critical for establishing whether EA is a mechanism of SAD symptoms. In particular, one key criterion for identifying treatment mechanisms is evaluating the timeline of the proposed mediator and outcome throughout treatment (Kazdin, 2009). According to Kazdin (2009, p. 424), to effectively evaluate the timeline of the proposed mediator and outcome: "(a) The proposed mediator must be assessed before the proposed outcome, and (b) the outcome must also be assessed early to ensure that the mediator has, in fact, changed before the outcome." To test this criterion, he recommends "session-by-session" assessment of both the mediator and outcome. To our knowledge, no prior studies have fulfilled this criterion with EA and SAD symptoms.

A related limitation is that longitudinal findings conflict in their support for whether changes in SAD symptoms lead to changes in EA or vice versa. To our knowledge, there is only prior study testing bidirectional change between EA and SAD symptoms over time, and no research evaluating this question in adults. Specifically, in a 3-wave cross-lagged path analysis collected at 4-month intervals, SAD symptoms predicted subsequently greater EA but not vice versa, among an unselected sample of middle school-aged adolescents (Shimoda, 2018). Broadening out a review to studies measuring general anxiety pathology, the findings are contradictory, with some longitudinal studies finding that EA unidirectionally predicts anxiety symptoms (Moroz \& Dunkley, 2019) and others finding that EA and stress/anxiety symptoms bidirectionally predict each other (Spinhoven et al., 2014). Importantly however, none of these prior studies tested a time-course that would map onto relations observed in a typical intervention (e.g., week-to-week fluctuations), or relations in the context of an intervention directly targeting one or both symptoms. Theoretically, it is critical to establish whether changes in SAD predict changes in EA across time - a question that has received disproportionally little attention. Clinically, mapping this time-course could help right-size clinical and patient expectations throughout an episode of care, which could improve motivation and preserve rapport.

To address these important limitations of prior research, the present study used bivariate latent change score modeling to model the bidirectional, dynamic relations between EA and SAD symptoms. Following Kazdin's (2009, p. 423) recommendations, we tested changes in EA and SAD symptoms as they unfolded session by session in response to a weekly, 3-session pilot intervention.

\section{Approach-Avoidance Task Training for EA}

Building on previous research, we developed a brief pilot intervention designed to shift EA. The intervention used in the present study consisted of psychoeducation about EA and an adaptation of the Approach-Avoidance Task (AAT) training, a type of cognitive bias modification. In the AAT, participants are trained to increase approach tendencies toward stimuli by pairing to-be-approached stimuli with an arm extension (i.e., pulling) that enlarges the stimuli on the screen, while pairing neutral or to-be-avoided stimuli with an arm flexion (i.e., pushing) that decreases the size of the image on the screen (Cacioppo et al., 1993). Supporting its efficacy, findings suggest that the AAT may be effective for improving SAD-related behaviors (e.g., intimacy building) and state affect (Loijen et al., 2021; Rinck et al., 2013; Taylor \& Amir, 2012; though see Asnaani et al., 2014).

The psychoeducation component of the intervention drew from acceptance-based therapies (e.g., Acceptance and Commitment Therapy; Hayes et al., 2006) and several studies that explicitly instructed participants to accept emotions using psychoeducation about emotional avoidance (Campbell-Sills et al., 2006; Hofmann et al., 2009; Wolgast et al., 2011). Acceptance (vs. control, suppression, or no instruction) has generally been associated with improved anxiety-related outcomes including lower state anxiety, desired avoidance (CampbellSills et al., 2006; Levitt et al., 2004), and physiological arousal (Hofmann et al., 2009) in response to stressor tasks. 


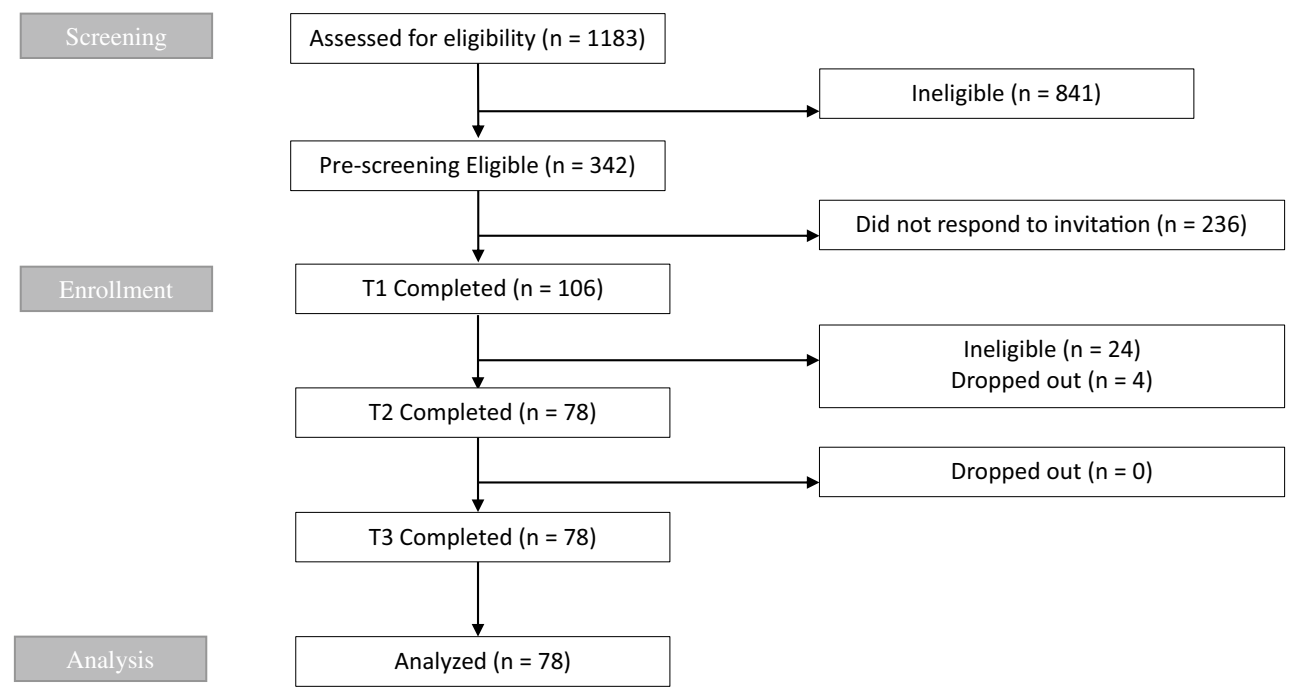

Fig. 1 Consort diagram

\section{Present Study}

The primary aim of the present study was to test the temporal course of EA and SAD symptoms by dynamically modeling whether reductions in EA temporally preceded and predicted reductions in SAD symptoms and/or vice versa from week to week. It was hypothesized that EA reductions would directly precede and predict SAD symptom reduction (Niles et al., 2014) and vice versa (Shimoda et al., 2018). Bivariate latent change score modeling, a type of structural equation modeling that accounts for each variable's overall trajectory of change and preceding measurement, was used to map the bidirectional, temporal course between EA and SAD symptoms. These dynamics were mapped from session to session of a 3-session pilot intervention designed to target EA. Note that a novel (vs. established) intervention without a control group was used, as we aimed to test feasibility and preliminary effects of this new intervention (not reported here). Piloting novel interventions before full trials aligns with best practice recommendations for development, evaluation, and implementation (Craig et al., 2008).

\section{Materials and Methods}

\section{Participants}

Participants were recruited from a Midwestern university. Informed consent was obtained from all participants. Interested students completed an initial screener to determine eligibility. Inclusion criteria at pre-screening included: 1) scores greater than one standard deviation below the mean on the Brief Experiential Avoidance Questionnaire
(BEAQ; Gamez et al., 2014) for an outpatient sample primarily diagnosed with an anxiety or depressive disorder (i.e., BEAQ scores $\geq 39.80$; Gamez et al., 2014); 2) scores at or higher than one standard deviation below the mean on the Social Phobia Inventory (SPIN; Connor et al., 2000) for an outpatient sample who met criteria for SAD (i.e., SPIN scores $\geq 25$; Campbell-Sills et al., 2015); 3) $\geq 18$ years of age; and 4) self-reported English fluency. The final sample included in analyses was limited to participants who still met inclusion criteria at Session 1 and completed all three sessions of the study $(N=78)$. See Fig. 1 for CONSORT diagram.

\section{Materials $^{1}$}

\section{Demographics}

Participants were asked to provide their age, gender, race, ethnicity, education attained, and written and verbal fluency in English. See Table 1 for demographics.

\section{EA}

The self-report BEAQ (Gamez et al., 2014) was used to assess trait levels of EA. In this measure, statements were rated on a scale from 1 (strongly disagree) to 6 (strongly agree) and included assertions such as "The key to a good life is never feeling any pain." This 15 -item measure has

\footnotetext{
1 The variables described and tested for the present study are part of a larger project. For a complete list of measures, please contact the first author.
} 
Table 1 Demographic and sample characteristics

\begin{tabular}{lll}
\hline & M or $\%$ & SD or N \\
\hline Age (years) & 19.42 & 2.29 \\
Gender & & \\
$\quad$ Female & $83.30 \%$ & 65 \\
$\quad$ Male & $16.70 \%$ & 13 \\
Race & & \\
$\quad$ Asian & $5.10 \%$ & 4 \\
Black/African American & $7.70 \%$ & 6 \\
White & $80.80 \%$ & 63 \\
$\quad$ Multiracial & $6.40 \%$ & 5 \\
Ethnicity & & \\
$\quad$ Hispanic/Latino & $2.60 \%$ & 2 \\
Not Hispanic/Latino & $97.40 \%$ & 76 \\
Experiential Avoidance & & \\
BEAQ1 & 58.13 & 9.06 \\
BEAQ2 & 53.54 & 11.16 \\
BEAQ3 & 48.06 & 12.97 \\
Social Anxiety Disorder Symptoms & & \\
SPIN1 & 38.26 & 8.92 \\
SPIN2 & 31.53 & 11.35 \\
SPIN3 & 25.23 & 12.78 \\
\hline
\end{tabular}

$B E A Q 1$ Brief Experiential Avoidance Questionnaire from Session 1, $B E A Q 2$ Brief Experiential Avoidance Questionnaire from Session 2, $B E A Q 3$ Brief Experiential Avoidance Questionnaire from Session 3, SPIN1 Social Phobia Inventory from Session 1, SPIN2 Social Phobia Inventory from Session 2, SPIN3 Social Phobia Inventory from Session 3

demonstrated good internal consistency, construct validity, convergent validity, and divergent validity (Gamez et al., 2014). In part because of this strong validity and reliability, the BEAQ has been recommended over other widely-used measures of EA (Rochefort et al., 2018; Tyndall et al., 2019). In the present study, internal consistency was adequate during each session (Cronbach's Alpha: Session $1=0.78$; Session $2=0.87$; Session $3=0.91$ ).

\section{SAD Symptoms}

The 17-item SPIN (Connor et al., 2000) was used to assess trait SAD symptoms. Items were rated on a 5-point Likert scale from 0 (not at all) to 4 (extremely). This measure has demonstrated good internal consistency, test-retest reliability, sensitivity to treatment effects, and convergent and divergent validity (Antony et al., 2006; Connor et al., 2000). In the present study, internal consistency was good during each session (Cronbach's Alpha: Session 1 =0.81; Session 2=0.89; Session 3=0.92).

\section{AAT Training Rationale and EA Psychoeducation}

The pilot intervention consisted of three sessions, based on evidence that a single AAT session can lead to meaningful change (e.g., Amir et al., 2013), though repeated administrations lead to greater changes (Eberl et al., 2014). During Session 1, participants received a rationale for the AAT training (detailed in the Online Resource), which is thought to increase engagement and buy-in (Lindgren et al., 2015). Further, they received psychoeducation about the benefits of emotional acceptance, the problematic effects of EA, and strategies to reduce EA (e.g., CampbellSills et al., 2006; Hayes et al., 2006). During Sessions 2 and 3, participants received a brief review of the training rationale and psychoeducation to reinstate the purpose of training. All training rationale and psychoeducation was provided in video format with accompanying handouts to enhance consistency. It included brief pauses during which research assistants asked participants to provide examples from their own lives (e.g., "What is an example of a time you have avoided your emotions?").

\section{AAT Training}

For the AAT training, participants viewed 60 emotionrelated words (e.g., anxious, happy) and, following Amir et al. (2013), 60 neutral words related to household objects (e.g., chair, dishwasher). A range of emotion words was included, because individuals with SAD may avoid both putatively positive and negative emotions (e.g., Farmer \& Kashdan, 2012; Kashdan \& Collins, 2010). Words were selected from the Affective Norms for English Words database (Bradley \& Lang, 1999). See Online Resource for stimuli. Participants were explicitly instructed to pull all emotion-related words toward themselves and push all household-related words away. These explicit instructions were based on meta-analytic findings that explicit instructions in AAT trainings are associated with greater effect sizes than instructions that attempt to conceal the purpose of the training by asking participants to focus on neutral aspects of the stimuli (e.g., push/pull based on the color or shape of the stimuli; Phaf et al., 2014). To help ensure that participants were engaging with the specifically selected stimuli, participants were presented with words (vs. pictures; Phaf et al., 2014) and were told to try to experience the emotion as they pulled the word toward them. As participants pulled and pushed, the images of the words were enlarged and minimized on the screen, respectively (Wiers et al., 2011). Each AAT block took approximately three to five minutes to complete. See Online Resource for additional details regarding the AAT methodology. 


\section{Procedure}

Participants came to the laboratory to complete the study. All three sessions were required to be completed within four weeks ( $M$ days between Sessions 1 and $2=8.12, S D=2.93$; $M$ days between Sessions 2 and $3=7.03, S D=2.01 ; M$ days between Sessions 1 and $3=15.23, S D=2.99)$. During each study visit, participants completed measures assessing SAD symptoms and EA. During Session 1, participants completed the measures, followed by the AAT rationale and psychoeducation. Last, they completed the AAT expectations questionnaire and the first AAT training. During Session 2, participants received the AAT rationale and psychoeducation, then completed the AAT training, followed by the measures. Session 3 was similar to Session 2, except that participants also completed the exit survey at the end of the session.

\section{Planned Analyses}

\section{Missing Data}

Four participants $(4.8 \%)$ chose not to continue after Session 1, and thus did not have data for Sessions 2 and 3. A nonsignificant Little's MCAR test with all variables collected during Session 1 and used in the present analyses suggested that the data were missing completely at random, $\chi^{2}=1.72, d f=3, p=0.63$. Thus, the four participants who did not complete Sessions 2 and 3 were dropped from the analyses, consistent with recommendations for small amounts of missing data (Kline, 2011).

\section{Temporal Course}

Bivariate latent change score modeling (BLCSM) was used to test the dynamic, temporal interrelations between changes in EA and changes in SAD symptoms. BLCSM has important advantages for testing this question, as it combines strengths of cross-lagged regression and growth curve modeling. Specifically, BLCSM models both time-precedence between variables and growth over time (Ferrer \& McArdle, 2010). Further, variables were modeled with latent scores to minimize error (Ferrer \& McArdle, 2010), and EA and SAD symptoms were measured at each timepoint of the intervention versus pre-post to better test the temporal course of their dynamics (Kazdin \& Nock, 2003). In BLCSM, modeling 'dynamic relations' refers to modeling whether change in one variable predicts change in the other, termed coupling, controlling for the both variables' previous measurements and overall trajectories of change (Ferrer \& McArdle, 2010). Together,
BLCSM provides a rigorous and focused model of possible causal relations between two variables. Thus, using this framework, we were able to optimally model whether longitudinal evidence was consistent with EA predicting changes in SAD symptoms and/or vice versa, from week-to-week.

Prior to testing the full BLCSM models, four preliminary univariate latent score models with EA and SAD symptoms were used to model the change processes subsumed within the full BLCSM model. This helped ensure that alternative, simpler models of change were not better ways of modeling change in EA and SAD symptoms than the full BLCSM model. The first univariate model tested a No Change Model, in which good fit would indicate that no change occurred in EA or SAD symptoms across time. The second tested a Proportional Change Model, which is conceptually similar to regression to the mean. Here, good fit would indicate that change in one variable could be adequately predicted by the previous latent score of the same variable, termed autoproportionality (represented by $\beta)$. The third tested a Constant Change Model, in which good fit would indicate that change in EA and/or SAD symptoms was best modeled by constant linear change, or constant slope (represented by $\alpha$ ), over time. The fourth was a Univariate Latent Change Model, which included both slope and autoproportionality, Here, a good-fitting model would indicate that change in one variable was best modeled by including both slope and autoproportionality for that same variable. The full BLCSM model incorporated the Univariate Latent Change Models of both EA and SAD symptoms. Incorporating both models allowed us to estimate the coupling parameter (represented by $\gamma$ ), or the portion of change in one variable that was directly predicted by the preceding latent change in the other variable. A positive $\gamma$ would indicate that changes in EA and SAD were in the same direction (e.g., decreases in one variable preceded and predicted decreases in the other). A negative $\gamma$ would indicate that the changes were in the opposite direction (e.g., increases in one variable preceded and predicted decreases in the other).

Models were fit using AMOS 25 estimated with default maximum likelihood. Model fit was evaluated using the comparative fit index (CFI), root mean square error of approximation (RMSEA), $\chi^{2}$ test, and $\chi^{2} / d f$ (Magee $\&$ Winhusen, 2016). Though there is some debate about cut-off values for fit statistics, the current rules of thumb are that good fit is indicated by CFI greater than 0.95, RMSEA less than 0.06, nonsignificant $\chi^{2}$ test (i.e., $p$-value greater than 0.05 ) and nonsignificant $\chi^{2} / d f$ values of 3 or less (Hoe, 2008; Hu \& Bentler, 1999; Weston \& Gore, 2006). When checking model fit with outliers removed, full maximum information likelihood was used to estimate missing values. Otherwise, there were no missing data to estimate. Following recommendations to estimate and 


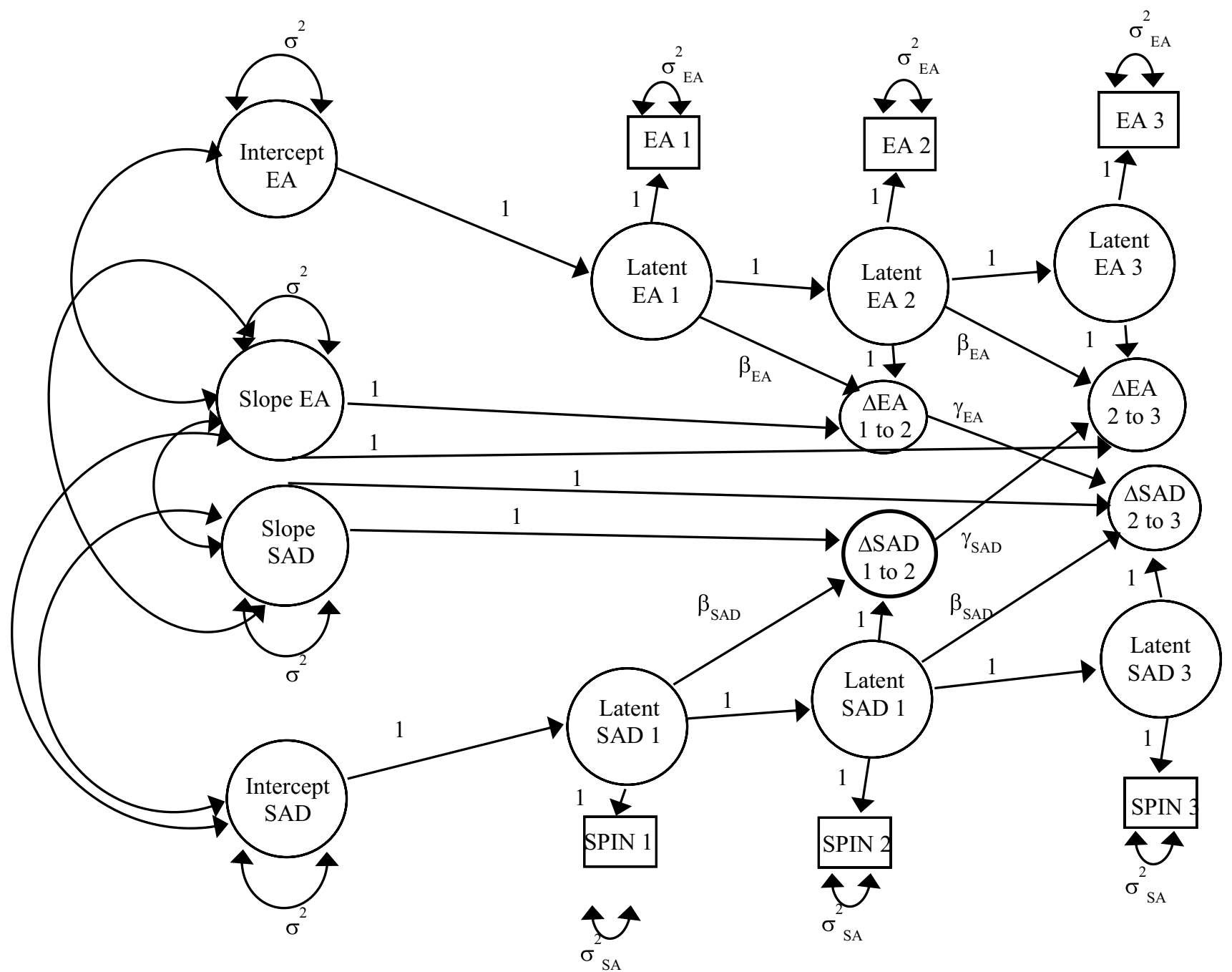

Fig. 2 Path Diagram of the Bivariate Latent Change Score Model for EA and SAD Symptoms. Note. Squares represent manifest variables, circles represent latent variables, single-headed arrows represent regression paths, and double-headed arrows represent variances $\left(\sigma^{2}\right)$ and covariances. Errors were covaried between the manifest variables that were measured across the same timepoint but are not represented in the figure for readability. $\gamma_{\mathrm{EA}}=$ coupling parameter with EA preceding and predicting change in SAD symptoms. $\gamma_{\mathrm{SAD}}=$ coupling parameter with SAD symptoms preceding and predicting change in EA. $\beta_{\mathrm{EA}}=$ autoproportionality of EA. $\beta_{\mathrm{SAD}}=$ autoproportionality of SAD symptoms. $\triangle$ EA 1 to $2=$ latent change in EA from Session 1 to Session 2. $\triangle$ EA 2 to $3=$ latent change in EA from Session 2 to Session 3. $\triangle \mathrm{SAD} 1$ to $2=$ latent change in SAD from Session 1 to Session 2. $\triangle \mathrm{SAD} 2$ to $3=$ latent change in SAD from Session 2 to Session 3 identify the model, all manifest variable intercepts were set to zero, and slope and autoproportionality were constrained to be constant across time (Magee \& Winhusen, 2016; McArdle, 2009). Errors were covaried between the manifest variables that were measured across the same timepoint to account for error that might be associated with a given timepoint. Similarly, the errors of the slopes and intercepts were correlated within and between EA and SAD symptoms (see Fig. 2). The datasets generated and analyzed for the current study are available from the corresponding author on reasonable request.

\section{Results}

\section{Did Change in EA Precede and Predict Change in SAD Symptoms and/or Vice Versa?}

\section{Univariate Latent Change Score Models}

The four preliminary models accounting for different types of univariate change were run in order of increasing complexity. As shown in Table 2, the No Change 


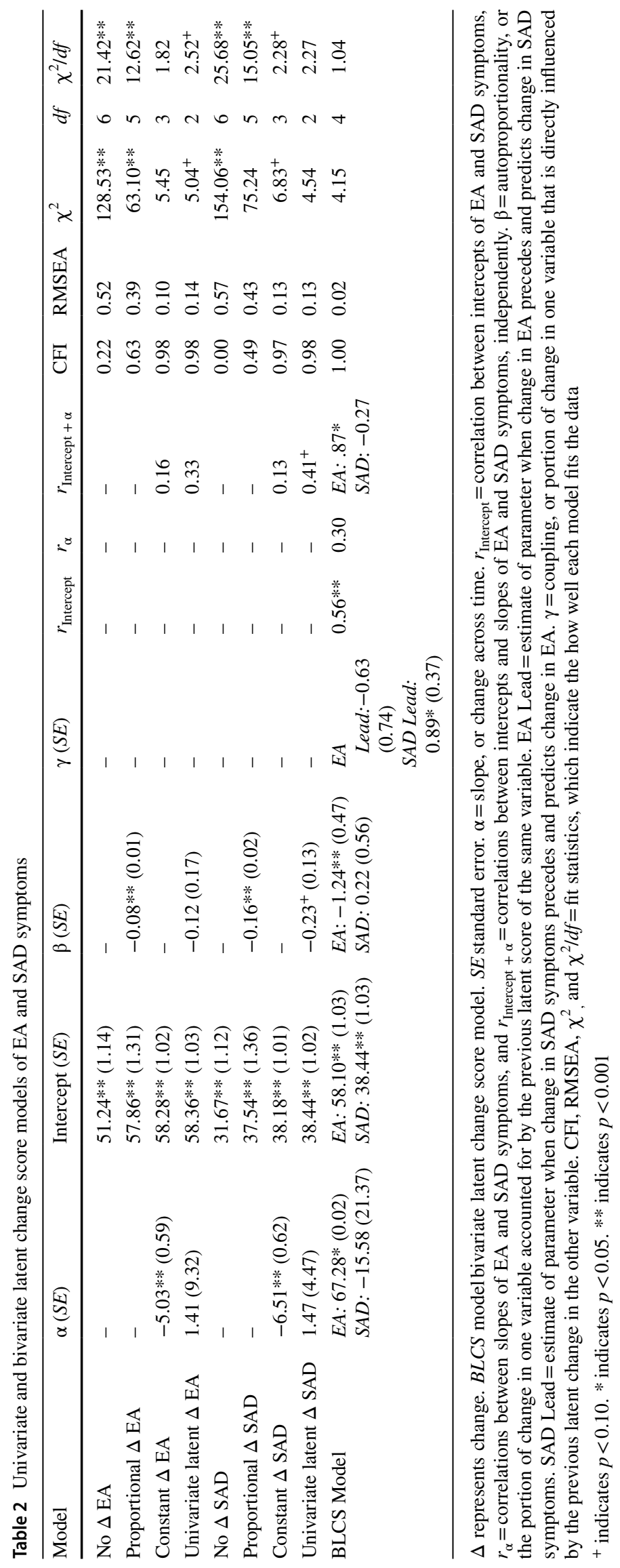


models' fit statistics suggested that these models should be rejected. The Proportional Change models also fit poorly. The Constant Change models fit slightly better, with both the EA and SAD symptom models demonstrating significant and negative slopes, suggesting linear decreases. Finally, the Univariate Latent Change Score models also demonstrated borderline fit statistics. Together, the Constant Change and Univariate Latent Change Score models suggested that modeling slope or both slope and autoproportionality, respectively, improved the fit by some statistics, but not others. Although these models appeared to fit better than No Change and Proportional Change, the overall patterns of fit indices indicated that there was still room for improvement.

\section{Bivariate Latent Change Score Models}

As demonstrated by the fit statistics in Table 2, the BLCSM model demonstrated the best fit to the data. With respect to our primary hypothesis of bidirectional coupling between EA and SAD symptoms, the coupling parameter with SAD symptoms preceding EA was significant and positive $(\gamma=0.89, p=0.02)$. This suggested that, controlling for slope and autoproportionality, weekly decreases in SAD symptoms predicted decreases in EA during the next week. Contrary to hypotheses, the coupling parameter with EA preceding SAD symptoms was not significant $(\gamma=-0.63, p=0.40)$, suggesting that weekly change in EA did not precede and predict weekly change in SAD symptoms.

In sum, when compared to the univariate models, the BLCSM model with EA and SAD symptoms demonstrated the best fit to the data. The coupling parameter suggested that weekly decreases in SAD symptoms predicted decreases in EA during the next week. This change was not bidirectional, as change in EA did not precede and predict change in $\mathrm{SAD}$ symptoms.

\section{Discussion}

The current project tested whether changes in experiential avoidance (EA) and social anxiety disorder (SAD) symptoms bidirectionally predicted one another from session to session. Despite the increasing ubiquity of targeting EA through empirically-supported treatments (e.g., Barlow et al., 2004; Hayes et al., 2006; Roemer et al., 2008), to our knowledge, this is the first study to directly test how EA and SAD symptoms may bidirectionally interrelate throughout an intervention-a key step in establishing EA as a mechanism of SAD (Kazdin, 2009). In partial support of hypotheses, weekly changes in SAD symptoms preceded and predicted changes in EA during the subsequent week. Surprisingly however, this effect was not bidirectional, as weekly changes in EA did not precede and predict changes in SAD symptoms during the subsequent week. Critically, these findings accounted for the overall trajectory of change in both variables (i.e., EA and $\mathrm{SAD}$ ) and both variables' preceding measurement, thereby eliminating two influential sources of potential covariation (i.e., important alternative explanations for these variables' interrelations).

Our novel findings beg the question, why might decreases in SAD symptoms precede and predict less avoidance of internal states? The measure of SAD used in the present study, the SPIN, may provide important clues. Factor analyses have identified three factors captured by the SPIN: fear of negative evaluation, fear of physical symptoms (i.e., akin to anxiety sensitivity), and fear of uncertainty (i.e., akin to intolerance of uncertainty) in social situations (CampbellSills et al., 2015). In other words, our findings suggest that reductions in fears of negative evaluation and uncertainty, as well as reductions in anxiety sensitivity, prospectively lead to reductions in self-reported EA.

Surprisingly, there is a dearth of research testing whether fears of negative evaluation, physical sensations, or uncertainty predict greater or less use of EA in individuals with SAD symptoms. However, indirect evidence suggests that each may predict EA in the context of the other disorders, such as symptoms of eating disorders (Espel-Huynh et al., 2019) and generalized anxiety disorder (Akbari \& Khanipour, 2018). Thus, building on the present study, a meaningful follow-up could explore how these constructs (i.e., fears of negative evaluation, physical symptoms, and uncertainty) work together to predict more or less use of EA for individuals with SAD symptoms. For example, prior research suggests that individuals with SAD symptoms fear that physical symptoms of anxiety will lead to negative evaluation (e.g., due to increased shaking, sweating, and blushing), thereby increasing the possibility of rejection (Kashdan \& Steger, 2006). Thus, we speculate that a natural, though often unhelpful, response is to use EA to try to eliminate physical and emotional indicators of anxiety, eliminating the possibility of rejection. However, if an individual with SAD symptoms blushes, but does not fear negative evaluation or can tolerate the possibility of being rejected, then there may be little reason to fear internal and physical sensations related to anxiety. In turn, there may be little need to use EA.

Contrary to predictions based on theoretical and empirical precedent, there was no evidence from this study that change in EA preceded and predicted change in SAD symptoms. This is the first study, to our knowledge, that followed recommendations to test session-by-session changes in both constructs to evaluate EA as a treatment mechanism of SAD symptoms (Kazdin, 2009). This finding was particularly 
notable, given that many empirically-supported treatments focus on directly targeting EA to reduce symptoms of psychopathology, including anxiety disorders (e.g., Barlow et al., 2004; Hayes et al., 2006; Roemer et al., 2008). This finding was also surprising in light of evidence that EA mediates treatment outcomes for SAD (Niles et al., 2014) and unidirectionally or bidirectionally predicts anxiety symptoms and stress (Moroz \& Dunkley, 2019; Spinhoven et al., 2014; Wenze et al., 2018).

There are a few possible explanations for our divergent pattern of findings. First, the present analyses controlled for the overall trajectory of change in both variables (i.e., EA and SAD) and both variables' preceding measurement, thus teasing apart sources of apparent change that may have influenced findings from past studies. In controlling for these sources, it is possible that our findings more accurately capture the relations between weekly fluctuations in SAD symptoms and EA, independent of longer-term trajectories or regression to the mean.

Second, none of the previous studies focused on the relations between EA and SAD symptoms within a weekly timeframe. Thus, when considering longer-term shifts over the entire course of therapy (e.g., 12 to 20 weeks), changes in EA may be an important mediator of changes in SAD symptoms (e.g., Niles et al., 2014). However, patients might not experience the long-term benefits of accepting their emotions until they have practiced for several weeks, learned to embrace the discomfort of emotions, or witnessed the benefit of emotional acceptance in their lives. If supported empirically with future research, mapping this longer time course may have important clinical utility. For example, a therapist might tell their patient that accepting difficult emotions will lead to lower SAD symptoms with practice, but the patient might not experience this change in symptoms for several weeks or even months - a caveat that could help manage patients' expectations about the pace of recovery.

\section{Limitations}

The findings from the present study must be considered in the context of a few key limitations. First, experts in structural equation modeling and BLCSM hold that sample sizes approaching 100 to 200 are generally preferable (Kline, 2011). That said, some have proposed that, "Smaller sample sizes are needed when the distributions of continuous outcome variables are normal in shape and their associations with one another are all linear" (Kline, 2011, p. 12), conditions that were met in the present study. Thus, we were approaching adequate sample size, but our promising findings should be replicated with a larger sample. Second, although our sample was selected for elevated SAD symptoms and EA, our findings may not generalize to individuals diagnosed with SAD or individuals seeking treatment for SAD. Third, because we used a pilot intervention without a control group, we cannot draw firm conclusions about the intervention's efficacy or the extent to which the findings reflect naturalistic versus manipulated interrelations between EA and SAD symptoms. Fourth, we used the BEAQ to evaluate changes in EA, because it has been recommended over other widely-used measures due its excellent construct, convergent, and discriminant validity (Rochefort et al., 2018; Tyndall et al., 2019). However, the BEAQ has been criticized for 1) using items related to behavioral (vs. internal) avoidance (Tyndall et al., 2019), and 2) demonstrating poor fit in certain samples (e.g., treatment-seeking veterans; Byllesby et al., 2020).

\section{Conclusions}

Despite these limitations, the present study is the first, to our knowledge, to test the bidirectional dynamic interrelations between EA and SAD symptoms as they unfold from week to week. Our findings suggest that changes in SAD symptoms precede and predict changes in EA, but not vice versa. This meaningful finding advances our understanding of how these constructs temporally interrelate throughout treatment. Moreover, they suggest that altering facets of SAD, such as fears of negative evaluation, physical sensations, and uncertainty, may have important downstream implications for reducing EA, representing an important area for future research. Critically, our findings also suggest that changes in EA do not necessarily lead to changes in SAD symptoms the following week. Given that many empirically-supported treatments target EA, this study highlights a need for future work to continue evaluating whether EA is indeed a mechanism of SAD symptom change.

Supplementary Information The online version contains supplementary material available at https://doi.org/10.1007/s10862-021-09899-8.

Acknowledgements Thank you to Dr. April Smith, Dr. Neil Brigden, and the ACE and SCOUT Labs for their invaluable feedback and support across the evolution of this project. Thank you to the research assistants for their hard work, accountability, and creativity in collecting our data (in alphabetical order): Chris Allen, Michael Archiable, Elise Ashford, Savannah Bliese, Alex Bronston, Robert Floyd, Christi Miley, Emma Kleymeyer, Yasamean Tadayon, and Isabelle Webber. This work was supported by funding provided to the first author from the Association of Behavioral and Cognitive Therapies, the Miami University Graduate School, and the Miami University Department of Psychology.

Authors' Contributions First author: conceptualization, methodology, software, formal analysis, investigation, data curation, writing - original draft, visualization, project administration, funding acquisition; Second author: methodology, software, validation, formal analysis, data curation, writing - review \& editing; Third author: conceptualization, 
methodology, software, validation, formal analysis, resources, writing - review \& editing, supervision.

Funding This work was supported by funding provided to the first author from the Association of Behavioral and Cognitive Therapies, the Miami University Graduate School, and the Miami University Department of Psychology.

Data Availability Data, code, and other materials will be made available upon request.

\section{Declarations}

Conflict of Interest Laurel D. Sarfan, Joshua C. Magee and Elise M. Clerkin have no conflicts of interest to disclose.

Experiment Participants All procedures performed in the study involving human participants were in accordance with the ethical standards of the institutional research committee and with the 1964 Helsinki declaration and its later amendments or comparable ethical standards.

Open Access This article is licensed under a Creative Commons Attribution 4.0 International License, which permits use, sharing, adaptation, distribution and reproduction in any medium or format, as long as you give appropriate credit to the original author(s) and the source, provide a link to the Creative Commons licence, and indicate if changes were made. The images or other third party material in this article are included in the article's Creative Commons licence, unless indicated otherwise in a credit line to the material. If material is not included in the article's Creative Commons licence and your intended use is not permitted by statutory regulation or exceeds the permitted use, you will need to obtain permission directly from the copyright holder. To view a copy of this licence, visit http://creativecommons.org/licenses/by/4.0/.

\section{References}

Akbari, M., \& Khanipour, H. (2018). The transdiagnostic model of worry: The mediating role of experiential avoidance. Personality and Individual Differences, 135, 166-172.

Amir, N., Kuckertz, J. M., \& Najmi, S. (2013). The effect of modifying automatic action tendencies on overt avoidance behaviors. Emotion, 13, 478-484. https://doi.org/10.1037/a0030443.The

Antony, M. M., Coons, M. J., Mccabe, R. E., Ashbaugh, A., \& Swinson, R. P. (2006). Psychometric properties of the social phobia inventory: Further evaluation. Behaviour Research and Therapy, 44, 1177-1185. https://doi.org/10.1016/j.brat.2005.08.013

Asnaani, A., Rinck, M., Becker, E., \& Hofmann, S. G. (2014). The effects of approach-avoidance modification on social anxiety disorder: A pilot study. Cognitive Therapy and Research, 38, 226-238.

Barlow, D. H., Allen, L. B., \& Choate, M. L. (2004). Toward a unified treatment for emotional disorders. Behavior Therapy, 35, 205-230.

Bradley, M. M., \& Lang, P. J. (1999). Affective norms for English words (ANEW): Instruction manual and affective ratings. Technical Report C-1. The Center for Research in Psychophysiology, University of Florida.

Byllesby, B. M., Stayton Coe, L. E., Dickstein, B. D., \& Chard, K. M. (2020). Psychometric evaluation of the Brief Experiential Avoidance Questionnaire among treatment-seeking veterans with posttraumatic stress disorder. Psychological Trauma: Theory, Research, Practice, and Policy, 12(7), 785-789.
Cacioppo, J. T., Priester, J. R., \& Berntson, G. G. (1993). Rudimentary determinants of attitudes. II: Arm flexion and extension have differential effects on attitudes. Journal of Personality and Social Psychology, 65(1), 5-17.

Campbell-Sills, L., Barlow, D. H., Brown, T. A., \& Hofmann, S. G. (2006). Effects of suppression and acceptance on emotional responses of individuals with anxiety and mood disorders. Behaviour Research and Therapy, 44, 1251-1263. https://doi.org/10. 1016/j.brat.2005.10.001

Campbell-Sills, L., Espejo, E., Ayers, C. R., Roy-Byrne, P., \& Stein, M. B. (2015). Latent dimensions of social anxiety disorder: A re-evaluation of the Social Phobia Inventory (SPIN). Journal of Anxiety Disorders, 36, 84-91. https://doi.org/10.1016/j.janxdis.2015.09.007

Chawla, N., \& Ostafin, B. (2007). Experiential avoidance as a functional dimensional approach to psychopathology: An empirical review. Journal of Clinical Psychology, 63, 871-890. https:// doi.org/10.1002/jclp.20400

Connor, K. M., Davidson, J. R. T., Churchill, L. E., Sherwood, A. W., Oa, E. F., \& Weisler, R. H. (2000). Psychometric properties of the Social Phobia Inventory (SPIN). British Journal of Psychiatry, 176, 379-386.

Craig, P., Dieppe, P., Macintyre, S., Michie, S., Nazareth, I., \& Petticrew, M. (2008). Developing and evaluating complex interventions: The new Medical Research Council guidance. $B M J, 337$, a 1655 .

Dalrymple, K. L., \& Herbert, J. D. (2007). Acceptance and Commitment Therapy for generalized social anxiety disorder. Current Opinion in Psychology, 31, 543-586. https://doi.org/10.1016/j. copsyc.2014.11.011

Eberl, C., Wiers, R. W., Pawelczack, S., Rinck, M., Becker, E. S., \& Lindenmeyer, J. (2014). Implementation of approach bias re-training in alcoholism-How many sessions are needed? Alcoholism: Clinical and Experimental Research, 38, 587-594. https://doi.org/10.1111/acer.12281

Espel-Huynh, H. M., Muratore, A. F., Virzi, N., Brookes, G., \& Zandberg, L. J. (2019). Mediating role of experiential avoidance in the relationship between anxiety sensitivity and eating disorder psychopathology: A clinical replication. Eating Behaviors, 34,101308 .

Farmer, A. S., \& Kashdan, T. B. (2012). Social anxiety and emotion regulation in daily life: Spillover effects on positive and negative social events. Cognitive Behaviour Therapy, 41, 152-162.

Ferrer, E., \& McArdle, J. J. (2010). Longitudinal modeling of developmental changes in psychological research. Current Directions in Psychological Science, 19(3), 149-154. https://doi.org/10. 1177/0963721410370300

Gamez, W., Chmielewski, M., Kotov, R., Ruggero, C., Suzuki, N., \& Watson, D. (2014). The brief experiential avoidance questionnaire: Development and initial validation. Psychological Assessment, 26, 35-45. https://doi.org/10.1037/a0034473

Glick, D. M., \& Orsillo, S. M. (2011). Relationships among social anxiety, self-focused attention, and experiential distress and avoidance. Journal of Cognitive and Behavioral Psychotherapies, 11(1), 1-12.

Hayes, S., Wilson, K., Gifford, E., Follette, V., \& Strosahl, K. (1996). Experiential avoidance and behavioral disorders: A functional dimensional approach to diagnosis and treatment. Journal of Consulting and Clinical Psychology, 64(6), 1152-1168.

Hayes, S. C., Luoma, J. B., Bond, F. W., Masuda, A., \& Lillis, J. (2006). Acceptance and commitment therapy: Model, processes and outcomes. Behaviour Research and Therapy, 44, 1-25. https://doi.org/10.1016/j.brat.2005.06.006

Heimberg, R. G., Hofmann, S. G., Liebowitz, M. R., Schneier, F. R., Smits, J. A. J., Stein, M. B., Hinton, D. E., \& Craske, M. G. (2014). Social Anxiety Disorder in DSM-5. Depression and Anxiety, 31, 472-479. 
Hoe, S. L. (2008). Issues and procedures in adopting structural equation modeling technique. Journal of Applied Quantitative Methods, 3, 76-83.

Hofmann, S. G., \& Bögels, S. M. (2006). Recent advances in the treatment of social phobia: Introduction to the Special Issue. Journal of Cognitive Psychotherapy: An International Quarterly, 20(1), 3-5.

Hofmann, S. G., Heering, S., Sawyer, A. T., \& Asnaani, A. (2009). How to handle anxiety: The effects of reappraisal, acceptance, and suppression strategies on anxious arousal. Behaviour Research and Therapy, 47, 389-394. https://doi.org/10.1016/j. brat.2009.02.010

Hu, L., \& Bentler, P. M. (1999). Cutoff criteria for fit indexes in covariance structure analysis: Conventional criteria versus new alternatives. Structural Equation Modeling, 6(1), 1-55.

Kashdan, T. B., \& Steger, M. F. (2006). Expanding the topography of social anxiety. Psychological Science, 17, 120-128.

Kashdan, T. B., \& Collins, R. L. (2010). Social anxiety and the experience of positive emotion and anger in everyday life: An ecological and momentary assessment approach. Anxiety, Stress, \& Coping, 23, 259-272.

Kashdan, T. B., Barrios, V., Forsyth, J. P., \& Steger, M. F. (2006). Experiential avoidance as a generalized psychological vulnerability: Comparisons with coping and emotion regulation strategies. Behaviour Research and Therapy, 44, 1301-1320. https:// doi.org/10.1016/j.brat.2005.10.003

Kashdan, T. B., Farmer, A. S., Adams, L. M., Ferssizidis, P., McKnight, P. E., \& Nezlek, J. B. (2013). Distinguishing healthy adults from people with social anxiety disorder: Evidence for the value of experiential avoidance and positive emotions in everyday social situations. Journal of Abnormal Psychology, 3, 645-655.

Kashdan, T. B., Goodman, F. R., Machell, K. A., Kleiman, E. M., Monfort, S. S., Ciarrochi, J., \& Nezlek, J. B. (2014). A contextual approach to experiential avoidance and social anxiety: Evidence from an experimental interaction and daily interactions of people with social anxiety disorder. Emotion, 14, 769-781. https://doi.org/10.1037/a0035935

Kazdin, A. E. (2009). Understanding how and why psychotherapy leads to change. Psychotherapy Research, 19, 418-428.

Kazdin, A. E., \& Nock, M. K. (2003). Delineating mechanisms of change in child and adolescent therapy: Methodological issues and research recommendations. Journal of Child Psychology and Psychiatry, 44, 1116-1129.

Kessler, R. C., Chiu, W., Demler, O., \& Walters, E. E. (2005). Prevalence, severity, and comorbidity of 12-month DSM-IV disorders in the national comorbidity survey replication. Archives of General Psychiatry, 62(6), 617-627. https://doi.org/10.1001/archpsyc. 62.6.617.Prevalence

Kessler, R. C., Cox, B. J., Green, J. G., Ormel, J., Mclaughlin, K. A., Merikangas, K. R., Petukhova, M., Pine, D. S., Russo, L. J., Swendsen, J., Wittchen, H. U., \& Zaslavsky, A. M. (2011). The effects of latent variables in the development of comorbidity among common mental disorders. Depression and Anxiety, 28, 29-39. https://doi.org/10.1002/da.20760.The

Kline, R. B. (2011). Principles and practice of structural equation modeling. The Guilford Press.

Levitt, J. T., Brown, T. A., Orsillo, S. M., \& Barlow, D. H. (2004). The effects of acceptance versus suppression of emotion on subjective and psychophysiological response to carbon dioxide challenge in patients with panic disorder. Behavior Therapy, 35(4), 747-766. https://doi.org/10.1016/S0005-7894(04)80018-2

Lindgren, K. P., Wiers, R. W., Teachman, B. A., Gasser, M. L., Westgate, E. C., Cousijn, J., Enkema, M. C., \& Neighbors, C. (2015). Attempted training of alcohol approach and drinking identity associations in us undergraduate drinkers: Null results from two studies. PLOS ONE, 10(8), 1-21. https://doi.org/10.1371/journal.pone.0134642
Loijen, A., Vrijsen, J. N., Egger, I. M., Becker, E. S., \& Rinck, M. (2021). Biased approach-avoidance tendencies in psychopathology: A systematic review of their assessment and modification. Clinical Psychology Review. https://doi.org/10.1016/j.cpr.2020. 101825

Magee, J. C., \& Winhusen, T. (2016). The coupling of nicotine and stimulant craving during treatment for stimulant dependence. Journal of Consulting and Clinical Psychology, 84, 230-237. https://doi.org/10.1037/ccp0000054

Mahaffey, B. L., Wheaton, M. G., Fabricant, L. E., Berman, N. C., \& Abramowitz, J. S. (2013). The contribution of experiential avoidance and social cognitions in the prediction of social anxiety. Behavioural and Cognitive Psychotherapy, 41, 52-65.

McArdle, J. J. (2009). Latent variable modeling of differences and changes with longitudinal data. Annual Review of Psychology, 60, 577-605. https://doi.org/10.1146/annurev.psych.60.110707. 163612

Moroz, M., \& Dunkley, D. M. (2019). Self-critical perfectionism, experiential avoidance, and depressive and anxious symptoms over two years: A three-wave longitudinal study. Behaviour Research and Therapy, 112, 18-27.

Niles, A. N., Burklund, L. J., Arch, J. J., Lieberman, M. D., Saxbe, D., \& Craske, M. G. (2014). Cognitive mediators of treatment for social anxiety disorder: Comparing acceptance and commitment therapy and cognitive-behavioral therapy. Behavior Therapy, 45(5), 664-677. https://doi.org/10.1016/j.beth.2014.04.006

Ossman, W. A., Wilson, K. G., Storaasli, R. D., \& McNeill, J. W. (2006). A preliminary investigation of the use of Acceptance and Commitment Therapy in group treatment for social phobia. International Journal of Psychology and Psychological Therapy, 6, 397-416.

O’Toole, M. S., Zachariae, R., \& Mennin, D. S. (2017). Social anxiety and emotion regulation flexibility: Considering emotional intensity and type as contextual factors. Anxiety, Stress, \& Coping, 30, 716-724.

Phaf, H. R., Mohr, S. E., Rotteveel, M., \& Wicherts, J. M. (2014). Approach, avoidance, and affect: A meta-analysis of approachavoidance tendencies in manual reaction time tasks. Frontiers in Psychology, 5, 1-16.

Rinck, M., Telli, S., Kampmann, I. L., Woud, M. L., Kerstholt, M., Velthuis, S., Wittkowski, M., \& Becker, E. S. (2013). Training approach-avoidance of smiling faces affects emotional vulnerability in socially anxious individuals. Frontiers in Human Neuroscience, 7, 1-6. https://doi.org/10.3389/fnhum.2013.00481

Rochefort, C., Baldwin, A. S., \& Chmielewski, M. (2018). Experiential avoidance: An examination of the construct validity of the AAQ-II and MEAQ. Behaviour Therapy, 49, 435-449.

Roemer, L., Orsillo, S. M., \& Salters-Pedneault, K. (2008). Efficacy of an acceptance-based behavior therapy for generalized anxiety disorder: Evaluation in a randomized controlled trial. Journal of Consulting and Clinical Psychology, 76, 1083-1089.

Shimoda, Y., Ishizu, K., \& Ohtsuki, T. (2018). The reciprocal relations between experiential avoidance and social anxiety among early adolescents: A prospective cohort study. Journal of Contextual Behavioral Science, 10, 115-119.

Spinhoven, P., Drost, J., de Rooij, M., van Hemert, A. M., \& Penninx, B. W. (2014). A longitudinal study of experiential avoidance in emotional disorders. Behavior Therapy, 45, 840-850.

Steinert, C., Hofmann, M., Leichsenring, F., \& Kruse, J. (2013). What do we know today about the prospective long-term course of social anxiety disorder? A systematic literature review. Journal of Anxiety Disorders, 27, 692-702.

Taylor, C. T., \& Amir, N. (2012). Modifying automatic approach action tendencies in individuals with elevated social anxiety symptoms. Behaviour Research and Therapy, 50, 529-536. https://doi.org/ 10.1016/j.brat.2012.05.004 
Tyndall, I., Waldeck, D., Pancani, L., Whelan, R., Roche, B., \& Dawson, D. L. (2019). The Acceptance and Action Questionnaire-II (AAQ-II). as a measure of experiential avoidance: Concerns over discriminant validity. Journal of Contextual Behavioral Science, $12,278-284$.

Wenze, S. J., Gaugler, T. L., Sheets, E. S., \& DeCicco, J. M. (2018). Momentary experiential avoidance: Within-person correlates, antecendents, and consequences and between-person moderators. Behaviour Research and Therapy, 107, 42-52.

Weston, R., \& Gore, P. A. (2006). A brief guide to structural equation modeling. The Counseling Psychologist, 34, 719-751.

Wiers, R. W., Eberl, C., Rinck, M., Becker, E. S., \& Lindenmeyer, J. (2011). Retraining automatic action tendencies changes alcoholic patients' approach bias for alcohol and improves treatment outcome. Psychological Science, 22, 490-497. https://doi.org/10. 1177/0956797611400615

Wolgast, M., Lundh, L. G., \& Viborg, G. (2011). Cognitive reappraisal and acceptance: An experimental comparison of two emotion regulation strategies. Behaviour Research and Therapy, 49, 858-866. https://doi.org/10.1016/j.brat.2011.09.011

Publisher's Note Springer Nature remains neutral with regard to jurisdictional claims in published maps and institutional affiliations. 